Технические науки

УДК 004.04

Коряков Игорь Витальевич

Начальник отдела разработки и производства технических средств ООО Научно-внедренческая фирма «Криптон»

\title{
ШИФРЫ КАК ФИЛЬТРЫ
}

Аннотация. Если представить иифры структурно в виде фильтров, в которых регистры с задержанными отсчётами будут хранить состояние иифра, а умножение на коэффициенты будет заменено некоторыми функииональными преобразованиями, то окажется, что шифр $S P N$ эквивалентен БИХ фильтру первого порядка, а шифр на основе сети Фейстеля - БИХ фильтру второго порядка с коэффициентом $a_{2}=1$. Причём вместо отсчётов входного сигнала на фильтр будет поступать последовательность подключей.

Обратимость канонической структуры фильтров (КСФ) позволяет построить класс шифров, в которых шифратор и дешифратор отличаются переменой мест функиий БИХ и КИХ ветвей. Такие шифры обладают рядом интересных свойств, описываемых ниже.

Ключевые слова: ичифровой фильтр, каноническая структура фильтра, криптография, блочный шифр, потоковый шифр.

\section{Цифровые фильтры}

Передаточная функция цифрового фильтра имеет вид [1, с.141]:

$$
H(z)=\frac{B(z)}{A(z)}=\frac{\sum_{m=0}^{M} b_{m} z^{-m}}{1-\sum_{n=1}^{N} a_{n} z^{-n}},
$$

где $z^{-t}$ - задержка сигнала на $t$ отсчётов, $b_{m}$ - коэффициенты нерекурсивной секции фильтра (фильтр с конечной импульсной характеристикой 
КИХ), $a_{n}$ - коэффициенты рекурсивной секции (фильтр с бесконечной импульсной характеристикой БИХ).

Разностное уравнение фильтра может быть представлено в форме:

$$
y(k)=x(k)-\sum_{n=1}^{N} a_{n} D_{n}(k-1)+\sum_{n=1}^{N} b_{n} D_{n}(k-1),
$$

где $y_{k}$ - выходной отсчёт фильтра, $x_{k}-$ входной отсчёт фильтра, $D_{n}()$ - значение на $n$-том выходе линии задержки, определяемой как

$$
D_{n}(k)=D_{n-1}(k-1) \mid n=N, \ldots, 2
$$

и

$$
D_{1}(k)=x(k)-\sum_{n=1}^{N} a_{n} D_{n}(k-1) .
$$

Каноническая структура фильтра (КСФ), соответствующая этому уравнению, может быть представлена в форме, приведённой на рисунке 1.

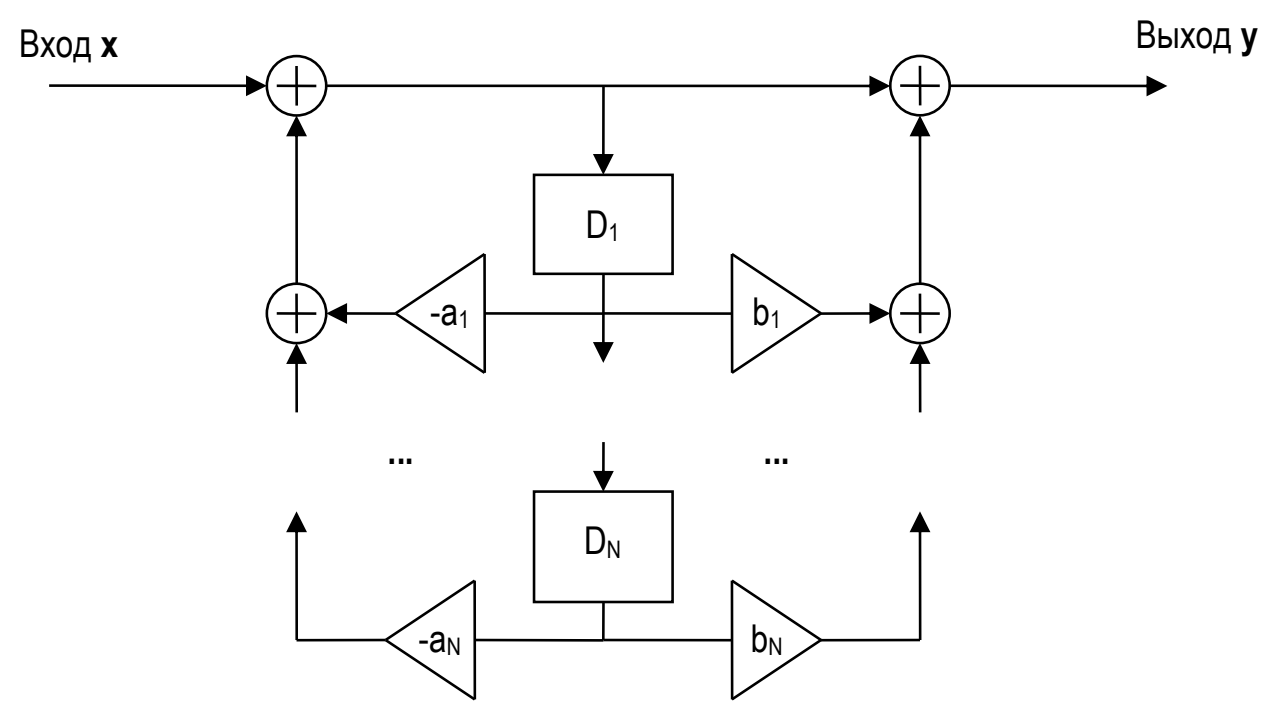

Рис. 1. Каноническая структура цифрового фильтра

\section{Сходство структур}

Существует множество разнообразных объектов с одинаковыми структурными признаками, аналоги деления или умножения на некий полином. Например, аналоги деления на полином:

- интегратор, 
- фильтр с бесконечной импульсной характеристикой (БИХ).

- самосинхронизирующийся скремблер,

- шифратор в режиме гаммирования с обратной связью (Cipher Feedback, CFB),

Объекты - аналоги умножения на полином:

- дешифратор в режиме CFB,

- самосинхронизирующийся дескремблер,

- дифференциатор,

- фильтр с конечной импульсной характеристикой (КИХ).

Децимирующий CIC фильтр (cascaded integral-comb, каскадный интегрально-гребенчатый фильтр) - сначала деление, затем умножение на полином.

Интерполирующий CIC фильтр - сначала умножение, затем деление на полином.

\section{Переход к структуре цифрового фильтра}

Традиционно принято изображать структуры блочных шифров в виде последовательности операций. Например, раунд шифра AES отображается в виде 4-х последовательных операций, изображённых на рисунке 2 в виде прямоугольников со стрелками:

1. Текущее состояние шифра $S_{i}$ является входом преобразования и подвергается табличной подстановке: SubBytes(state);

2. Перестановка: ShiftRows(state);

3. Перестановка: MixColumns(state);

4. Сложение с подключём: AddRoundKey(state) образует следующее состояние шифра $S_{i+1}$. 


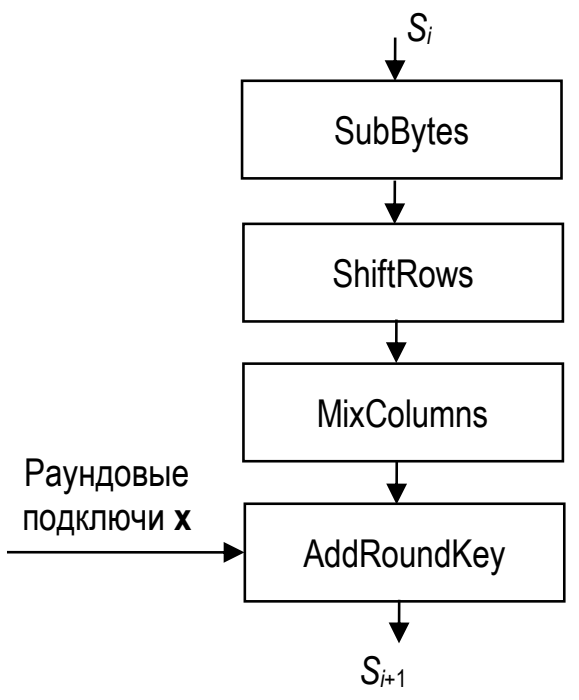

Рис. 2. Структура раунда шифра AES

A теперь посмотрим, что собой представляет шифр AES с точки зрения цифрового фильтра. Он содержит некий регистр состояния (128 бит), некое преобразование подстановки-перестановки $F$ и сложение с подключом $k_{r}$.

Итеративный блочный шифр типа SPN (substitution-permutation network), к которому относится стандарт AES, можно представить структурой БИХ фильтра с делением на полином. На рисунке 3 представлена структура БИХ фильтра первого порядка с передаточной функцией

$$
H(z)=\frac{1}{A(z)}=\frac{1}{1-a_{1} z^{-1}} \text {. }
$$

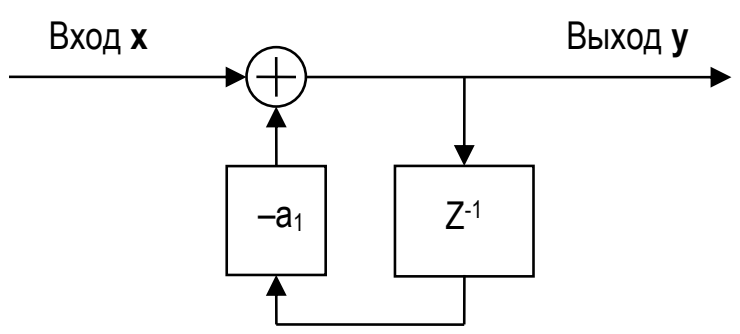

Рис. 3. Структура БИХ фильтра 1-го порядка 
Здесь $Z^{-1}$ - элемент задержки на один отсчёт (обычно параллельный регистр), $-a_{1}-$ умножитель на коэффициент $a_{1},+-$ сумматор. На вход фильтра поступают отсчёты сигнала $x$, на выходе фильтра формируются отсчёты $y$.

Теперь рассмотрим в общем виде структуру шифра SPN, представленную на рисунке 4.

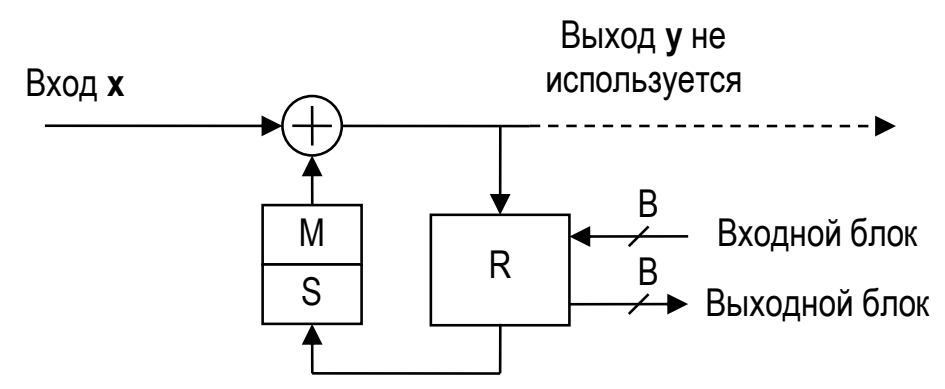

Рис. 4. Структура SPN шифратора

Здесь $\mathrm{R}$ - параллельный регистр, хранящий текущее состояние шифра В бит, S и M - раундовая функция (подстановка и перестановка, соответственно), + - сумматор. Если проводить аналогию с БИХ фильтром, то вместо умножителя стоит функциональный преобразователь, на вход фильтра поступают подключи $x$, начальное состояние - это входной блок, конечное состояние после некоторого числа тактов - результирующий блок, а выход фильтра $y$ не используется.

На рисунке 5 представлен БИХ фильтр второго порядка с передаточной функцией

$$
H(z)=\frac{1}{A(z)}=\frac{1}{1-a_{1} z^{-1}-a_{2} z^{-2}} .
$$




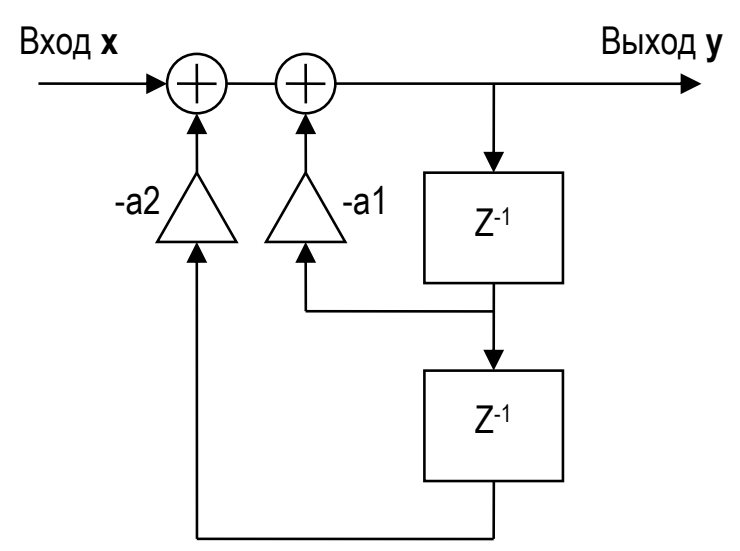

Рис. 5. Структура БИХ фильтра 2-го порядка

Здесь изображён фильтр 2-го порядка с двумя элементами задержки $Z^{-1}$ и двумя постоянными коэффициентами $-a_{1}$ и $-a_{2}$.

Это фактически схема Фейстеля, реализованная в ГОСТ 28147 (см. рисунок 6), у которой коэффициент $-a_{2}=1$, a $-a_{1}$ представлен последовательными преобразованиями $\mathrm{S}$ (узлы замены) и $\mathrm{M}$ (циклический сдвиг).

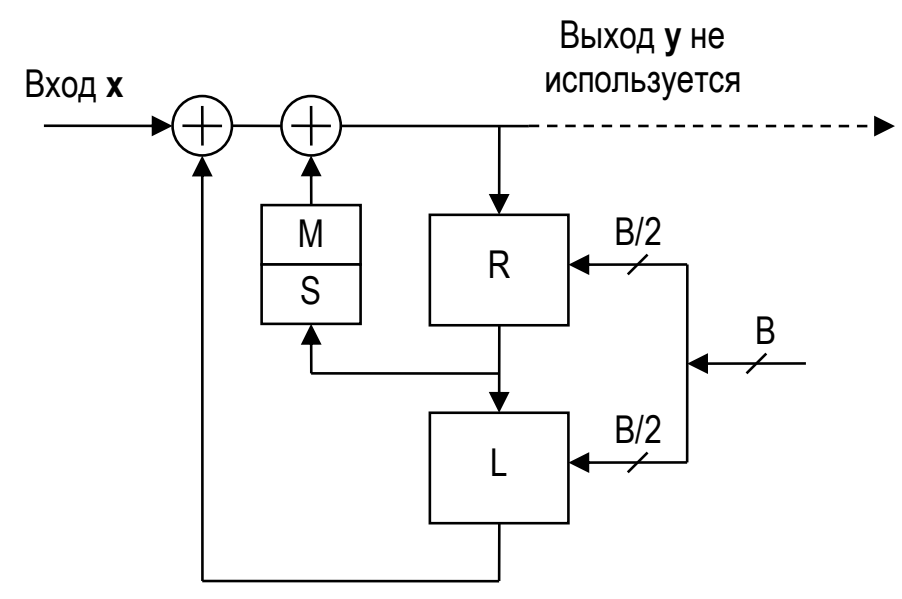

Рис. 6. Сеть Фейстеля как фильтр 2-го порядка

При этом входом является не сигнал $x$, а состояние элементов задержки $\mathrm{R}$ и $\mathrm{L}$ как правой и левой частей шириной $\mathrm{B} / 2$ входного блока шириной $\mathrm{B}$, а $x$ является последовательностью подключей. Выход фильтра $y$ не используется. Выходом преобразования является состояние элементов 
задержки после фильтрации $r$ отсчётов (число раундов) входного вектора $x$. На примере ГОСТ 28147 вход В составляет 64 разряда, регистры R и L 32-х разрядные, $\mathrm{S}$ - это восемь 4-х разрядных таблиц, М - циклический сдвиг. Подключи - это 32 целых 32-х разрядных отсчёта. Сложение с подключами делается в несколько другой точке (не после задержки в регистре $\mathrm{R}, \mathrm{a}$ до неё), место этой точки не влияет на свойства шифра.

Рассмотрим ещё один БИХ фильтр с двумя единичными коэффициентами $a_{i}=1$ и $a_{j}=1$ и остальными коэффициентами, равными нулю.

Такой фильтр будет иметь один полюс, расположенный на единичной окружности в $z$-плоскости и будет неустойчивым, то есть его импульсная характеристика не будет убывать. Реализация фильтра в виде линии задержки, на вход которой поступает сумма отводов $i$ и $j$ и будет представлять собой генератор при ненулевом начальном состоянии ЛЗ. Достаточно быстро такой генератор (при обычной целочисленной арифметике без насыщения) выйдет из линейного режима и будет переполняться по модулю разрядности арифметики. Если $i=24$ и $j=55$, то получится один из вариантов задержанного генератора Фибоначчи, широко применяемого в криптографии.

\section{Обратимость канонической структуры}

Для фильтра, определяемого в соответствии с формулой (1), при условии $N=M$ и $b_{0}=1$, возможен обратный фильтр с заменой местами числителя и знаменателя:

$$
H^{-1}(z)=\frac{A(z)}{B(z)}=\frac{\sum_{n=1}^{N} a_{n} z^{-n}}{1-\sum_{m=1}^{M} b_{m} z^{-m}} .
$$

Обратный фильтр по выходной последовательности $y$ прямого фильтра точно восстанавливает его входную последовательность $x$.

Свойство обратимости КСФ (восстановления входного сигнала по выходному) при перестановке коэффициентов $a_{n}$ и $b_{n}$ сохраняется, даже 
если сложение заменить любой коммутативной операцией, а умножение любым функциональным преобразованием, в том числе - необратимым.

На основе КСФ возможно построение шифра, в котором схемы зашифрования и расшифрования будут отличаться переменой мест преобразований $a_{n}$ и $b_{n}$.

Если мы зафиксируем размер внутреннего состояния шифра (например, 128 бит), то могут быть масштабируемыми порядок фильтра $N$ и ширина шифра $B$ (разрядность регистров линии задержки). Вплоть до $N=1$ и $B=128$ и до $N=128$ и $B=1$. Например, если мы задаём элемент, подвергающийся шифрованию, как байт, то получим шифр с $N=16$ и $B=8$.

При этом, в определённом смысле, $N$ является аналогом числа раундов блочного шифра, а преобразования $a_{n}$ и $b_{n}$ - аналогом раундовых функций.

И если для $B=1$ функции задаются простейшими подстановками: значение входа, инверсное значение входа, константа «0» и константа «1» (это приводит к классическим потоковым шифрам с одноразрядными регистрами), то для $B=8$ наиболее разумными представляются фиксированные случайные перестановки 8 х 8, заданные таблично, а для $B=128$ - вопрос требует особого изучения.

Рассмотрим конкретный вариант КСФ шифра с $N=1$ и $B=128$, схема зашифрования и расшифрования которого приведены на рисунке 7.

Здесь $\mathrm{D}_{1}$ - элемент задержки в виде 128-ми разрядного параллельного регистра, $a_{1}$ и $b_{1}$ - функциональные преобразования, приближённые к фиксированным случайным подстановкам 128 х 128. Операции сложения представлены 128-разрядными сумматорами по модулю 2. 


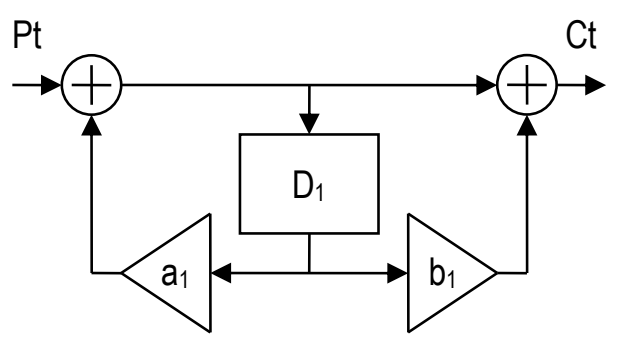

Зашифрование

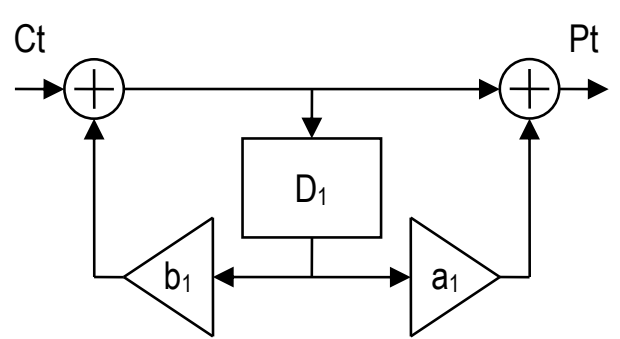

Расшифрование

Рис. 7. Схема КСФ шифра с $N=1$ и $B=128$

Зависимость преобразований от ключа может быть введена либо в начальное состояние (как в потоковых шифрах), либо в функциональные преобразования, например, как представлено на рисунке 8.

При этом ключ $\mathrm{K}$ длиной 256 бит разделяется на две половины $\mathrm{K}_{1}$ и $\mathrm{K}_{2}$, которые складываются по модулю 2 с входами и выходами функциональных преобразователей $a_{1}$ и $b_{1}$ в соответствии с шифром Ивен-Мансура [2] с доказуемой стойкостью.

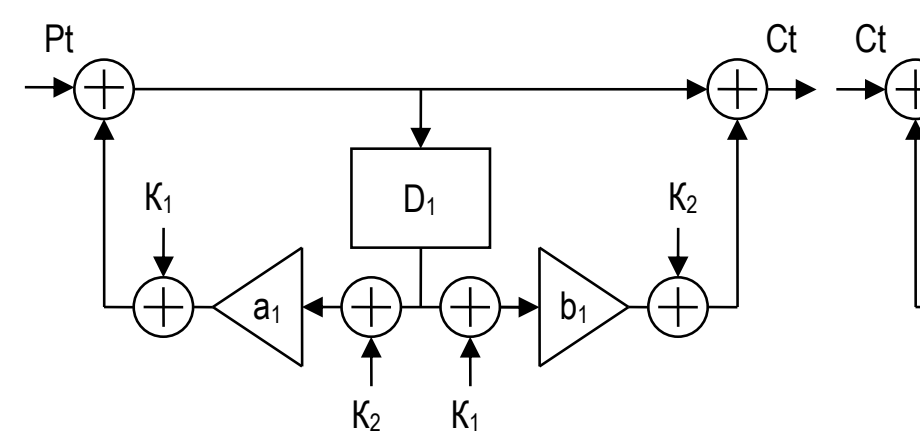

Зашифрование

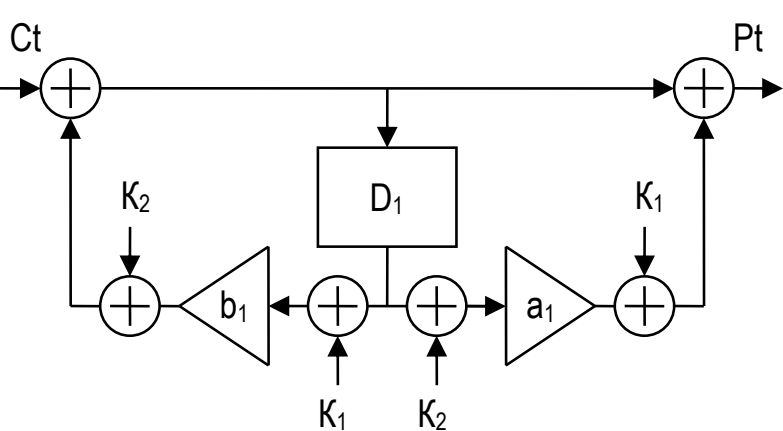

Расшифрование

Рис. 8. Пример схемы ввода подключей в функциональные преобразования

Функциональные преобразователи $a_{1}$ и $b_{1}$ могут быть реализованы в виде «широких» S-блоков, содержащих, например, по 16 таблиц фиксированных случайных подстановок $\mathrm{S}_{0}-\mathrm{S}_{15}$ размером 8 х 128, конкатенация 16-ти входов которых образует 128-ми разрядный вход преобразования, а сумма по модулю 2 всех 16-ти 128-ми разрядных выходов таблиц образует 
выход преобразования. Структура таких функциональных преобразователей приведена на рисунке 9.

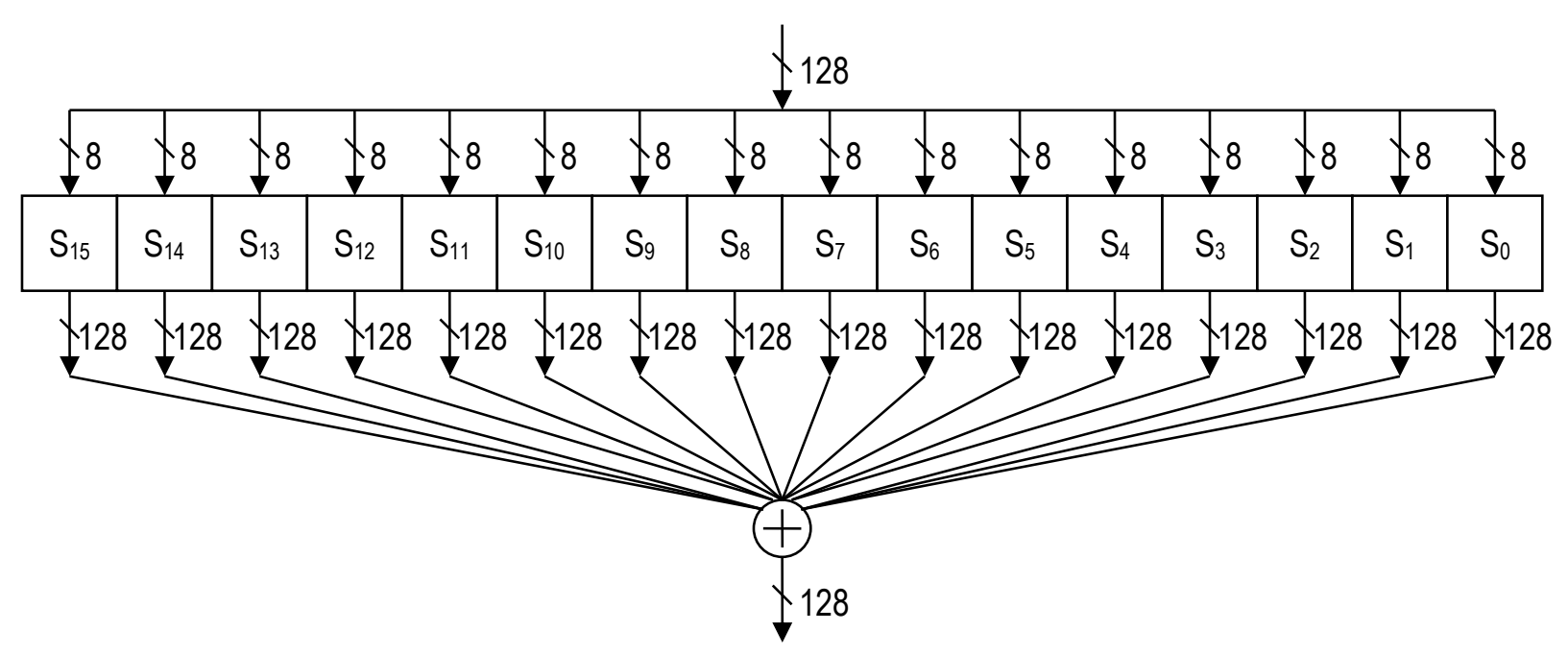

Рис. 9. Схема «широкого» S-блока

Какой-либо отбор S-блоков скорее всего не потребуется, поскольку комбинаторная мощность такого блока очень велика (2 в степени порядка $500000)$ и вероятность получения случайным образом «плохого» блока исчезающе мала. Такие S-блоки при шифровании $N^{*} B$ бит применяются однократно, в то время как в SPN шифрах один S-блок применяется повторно сотни раз при шифровании одного блока данных, что требует очень строгого подхода к его формированию. Конечно, объём памяти для реализации «широких» S-блоков довольно значителен - 128К байт для конкретного примера, но в настоящее время такой объём является вполне доступным pecyрсом.

Предполагаемым преимуществом класса шифров КСФ будет являться монотонная масштабируемость по $N$ и $B$, что помимо адаптации шифра к конкретным задачам позволит исследовать уменьшенные версии шифров. Кроме того, аппаратная реализация шифра позволяет сократить время шифрования одного блока до одного такта. 


\section{Литература}

1. Оппенгейм А. В., Шафер Р. В. Цифровая обработка сигналов. М.: Связь, 1979. 416 с.

2. Even S., Mansour Y. A Construction of a Cipher From a Single Pseudorandom Permutation. In: Imai, H., Rivest, R.L., Matsumoto, T. (eds.): ASIACRYPT 1991. LNCS, vol. 739. Springer, Heidelberg. 1993. P. 210224. 